\title{
Facial Expressions Reconstruction of 3D Faces based on Real Human Data
}

\author{
Jacey-Lynn Minoi, Amelia Jati Robert Jupit \\ Faculty of Computer Science \\ and Information Technology \\ Universiti Malaysia Sarawak \\ 94300 Kota Samarahan \\ Sarawak, Malaysia \\ Email: (jacey; rajati)@ fit.unimas.my
}

\author{
Duncan Fyfe Gillies \\ Department of Computing \\ Imperial College London \\ 180 Queen's Gate, \\ London SW7 2RH, UK \\ Email: d.gillies@imperial.ac.uk
}

\author{
Sylvester Arnab \\ Serious Game Institute \\ Coventry University Technology Park \\ Innovation Village \\ Cheetah Road \\ Coventry CV1 2TL \\ Email: sarnab@cad.coventry.ac.uk
}

\begin{abstract}
This paper presents an approach to reconstruct facial expressions using real data sets of people acquired by three-dimensional (3D) scanners. The acquired raw human face surfaces are pre-processed and a statistical shape model of the human face is built using multivariate statistical approaches. Our idea of using tensor model on the multivariate statistical method is to use all the face features found in the training set, with a variety of facial variations simultaneously by separating them into a number of classes. Point-to-point correspondences between the face surfaces are required in order to do the reconstruction processes. The advantage with the tensor-based multivariate statistical method is that it is practical to generate a variety of face shapes applied in different degrees, which would give a continuous and natural transition between the facial expressions. Our experiments focused on dense correspondence to compute the deformation of facial expressions. We have also used some selected landmark points placed on the face surfaces to compute the deformation of facial expressions. The selected landmark points are based on the Facial Action Coding System (FACS) framework and the movements are analysed according to the motion of the facial features. Besides altering human facial expressions, the presented approach could also be used to neutralise facial expression to aid the performance of face recognition.
\end{abstract}

\section{INTRODUCTION}

Facial expression reconstruction is a complex and difficult area of work which has started more than three decades ago. A large number of facial modelling and facial expression reconstruction works have employed feature-based deformation [1], [2], [3] and muscle-based approaches [4], [5], [6]. Feature-based approaches used facial features that contribute most to facial expressions, such as the eyelids, eyebrows, mouth, wrinkles and budges. The motions flow of features are measured and then used to animate facial expressions. Musclebased approach mimics the contractions of facial muscles. The combinations of the facial muscles are defined by Action Units (AUs) from the Facial Action Coding System (FACS) framework. Using AUs could reduce the amount of work in characterising facial expression data. Many face animators, for example [7], imitate facial muscles movements to generate facial expressions.

Multi-layer approach is another method to reconstruct facial expressions and is built from the anatomical structure of the face, facial muscles, soft tissues, skin and etc. [5], [6], [8]. This approach improves the realism of the facial expressions reconstruction, however the use of the sophisticated biomedical models requires accurate simulation methods and high computational costs [6]. Furthermore, animating expressions in complex multi-layer structures require non-linear methods to simulate dynamic deformation of the skin. Failing to create a detailed skin deformation (such as wrinkles) may results in less realistic facial expressions [9].

The geometry warping approach is another method for synthesizing facial expressions. The facial expression information is measured from two images - one with a neutral expression and another with a particular facial expression. The calculated facial movement difference vectors are transferred to a target image of a neutral face [3], [10], [11], [12]. The facial movement differences can be controlled by using linear interpolation methods. The disadvantage of this approach is that the overall shapes of the face, including geometric shapes, poses and orientation, and facial expressions, are calculated and computed together. It is therefore not a perfect solution for generating in-between expressions [9]. Recent work has been undertaken to overcome this weaknesses by using nonlinear interpolation or by combining linear and non-linear interpolation.

[11] used simple geometric interpolation to synthesize expression on 3D face models. The feature points are manually digitised on each face model. This was followed by automatic expression synthesis where the data of real actors are captured and analysed [12], [13], [14]. The captured face surfaces are represented using a structured mesh, along with texture information.

Segmenting face models into smaller regions is also employed with the aim of synthesizing only the relevant parts of the face contributing to an expression. [15] applied this approach on 3D face models. [16] employed a morphable technique to animate facial expressions on existing 2D images and videos. The advantage of the morphable modelling approach is that it can work on faces without acquiring examples of facial expression data of a person.

[17] mapped facial movements from a recorded video to 